\title{
Identification of Littoral Targets with a Laser Range Profiler
}

\author{
Johan C. van den Heuvel, Herman H.P.Th. Bekman, Frank J.M. van Putten, Leo H. Cohen \\ TNO Defence, Security and Safety, Oude Waalsdorperweg 63, The Hague, The Netherlands
}

\begin{abstract}
Naval operations in the littoral have to deal with threats at short range in cluttered environments with both neutral and hostile targets. On board naval vessels there is a need for fast identification, which is possible with a laser range profiler. Additionally, in a coast-surveillance scenario a laser range profiler can be used for identification of small sea-surface targets approaching the coast. An eye-safe 1.5 um laser range profiler has been used to validate these claims. Experimental results show that range profiles of sea-surface targets can be obtained at ranges of several km's. Seasurface clutter is shown to be negligible. Simulation shows that sea-surface targets can be distinguished from their range profiles. The influence on the identification performance of range resolution and a-priori knowledge of the aspect angle is presented. Classification has been tested on simulated range profiles of a number of small boats. With a range resolution of 0.3 meter (comparable to our experimental set-up), these small boats could be identified.
\end{abstract}

Keywords: Laser, littoral, range profile, identification

\section{INTRODUCTION}

Maritime borders and coastal zones are susceptible to threats like drug trafficking, piracy, undermining economical activities, etc. Effective surveillance of maritime borders and coastal zones is a necessity when they are subject to such threats. A laser range profiler can provide identification capability in combination with a detection sensor like radar. The detection sensor gives the location of the threat, while the laser range profiler can identify the target based on high range resolution data.

A similar application of a laser range profiler is in combination with an Infrared Search and Track (IRST) sensor on board of a naval vessel. Here the profiler adds identification capability of sea and air targets to the detection capability of an IRST. In addition, the profiler gives the range and can discriminate between false alarms and potential threats. False alarms that can limit IRST usefulness like birds, cloud edges, and sun glints can be easily discriminated from real targets by a laser range profiler.

A laser range profiler has the favorable properties of a good range resolution in combination with low sea-surface clutter. These properties have been validated by experiments with an eye-safe 1.5 um laser range profiler. A range resolution of $0.6 \mathrm{~m}$ has been achieved and $0.3 \mathrm{~m}$ (used in the simulation) is possible with deconvolution. Sea-surface reflection was negligible. Identification with laser range profiles is in principle easier than for radar range profiles since radar returns depend very strongly on target aspect angle due to the predominant specular reflection ${ }^{1,2}$ compared to the mainly-diffuse laser reflection.

In this paper we focus on improving identification results for small sea-surface targets in coast surveillance. For this purpose, a scenario for surveillance of the coastal environment has been created. On basis of simulated data for the profiler improved capabilities for recognizing and identifying non-cooperative ships in coastal waters are demonstrated, so that the quality of the maritime surface picture in the littoral environment can be enhanced. The scenario is as follows: the radar system detects and tracks ships, while simultaneously information from the laser range profiler is extracted. On basis of the comparison of the laser range profile (LRP) with an LRP in a database, the ship is identified.

Laser Radar Technology and Applications XII, edited by Monte D. Turner, Gary W. Kamerman, Proc. of SPIE Vol. 6550, 65500R, (2007) · 0277-786X/07/\$18 · doi: 10.1117/12.719279 


\section{SCENARIO}

The following scenario is defined. Sensors are located on a tower with a height of 40 meter and with a distance of 100 meter from the coast line. A ship is approaching the coast with a speed of 10 knots and with an angle of 75 degrees to the coast (see figure 2.1). The situation is at night which excludes the use of a visual camera. We consider the atmospheric conditions as optimal.
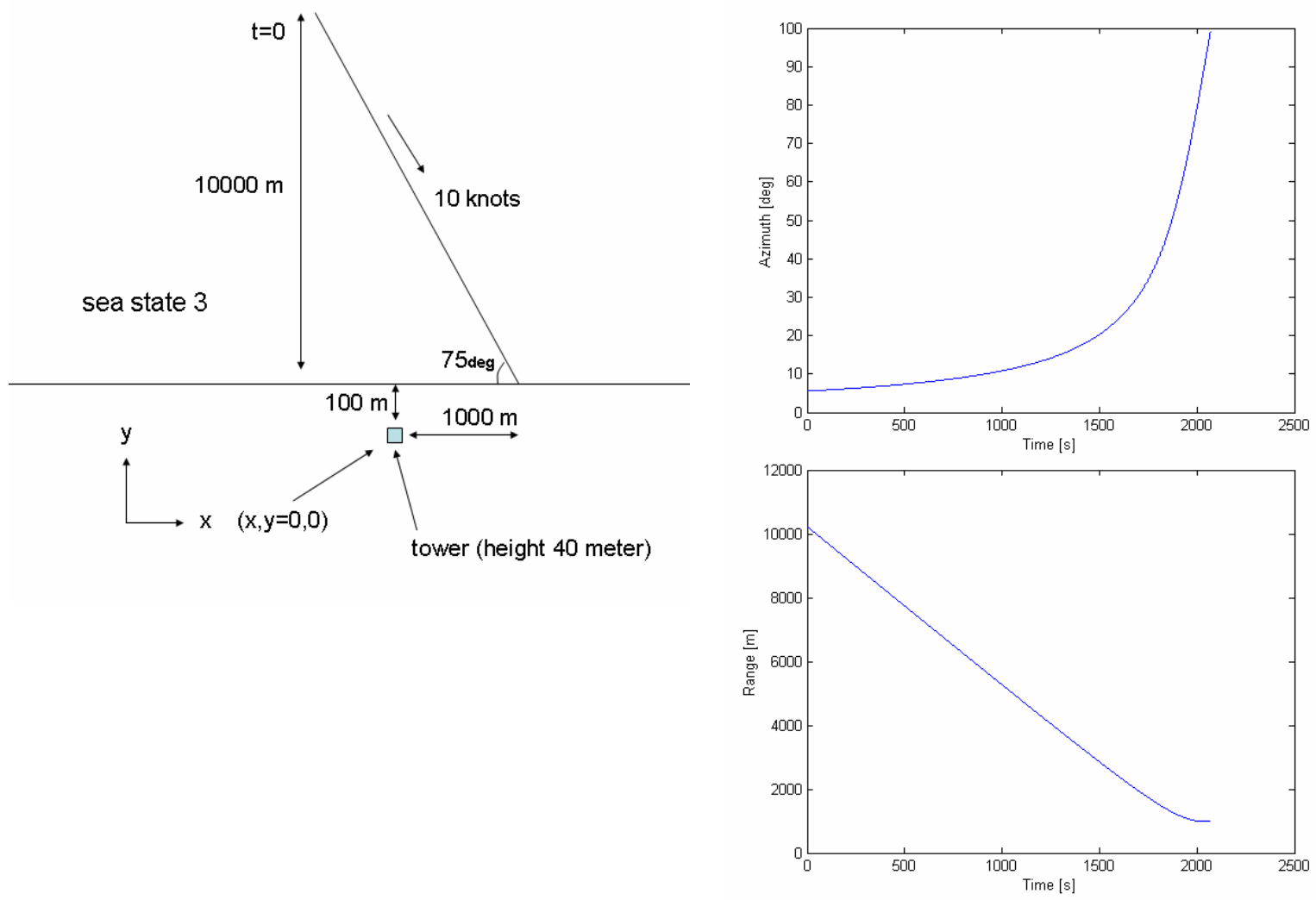

Figure 1 Layout of scenario with target range (bottom right) and target aspect angle (top right).

The scenario is for shallow waters like in the North Sea. A sea state category three is chosen which implies wave heights in the order of one 1 meter, wavelengths in the order 70 meter and periods of 7-10 seconds. These waves force floating platforms like ships to have periodically varying pitch, yaw and roll angles. We assume here no yaw, since yaw variation was negligible. Pitch and roll angles are in the order of a few (2-4) degrees. The pose of the platform is determined by an azimuth and elevation angle.

For the identification study, laser range profiles are simulated based on the target range and aspect angle in the scenario. The simulation of the laser range profiles is based on currently available technology with a range resolution of $30 \mathrm{~cm}$. The next section shows such a system and some relevant results are shown there.

\section{EXPERIMENTAL SET-UP}

An experimental laser range profiler was used to validate the usefulness of such a system for sea-surface target identification. The system is depicted in Figure 2. The green box contains the OPO laser wavelength converter, which converts the pump laser wavelength of $1.064 \mu \mathrm{m}$ into the more eye-safe wavelength of $1.57 \mu \mathrm{m}$. The white box on top of the green box contains the detector and receiver telescope. The white box right next to the green box is the 1.064 pump laser. 
The pump laser for the OPO is a Q-switched Nd:YAG laser. The maximum output is $350 \mathrm{~mJ}$ per pulse with a pulse length of $5 \mathrm{~ns}$ at a rep-rate of $20 \mathrm{~Hz}$. In the OPO the laser radiation at $1.064 \mu \mathrm{m}$ is converted to $1.57 \mu \mathrm{m}$. The conversion takes place in a non-linear crystal. To increase the non-linear conversion efficiency it is beneficial to increase the laser power density by focusing the laser beam into the crystal. However there is a limit on the maximum power density that the crystal can withstand.

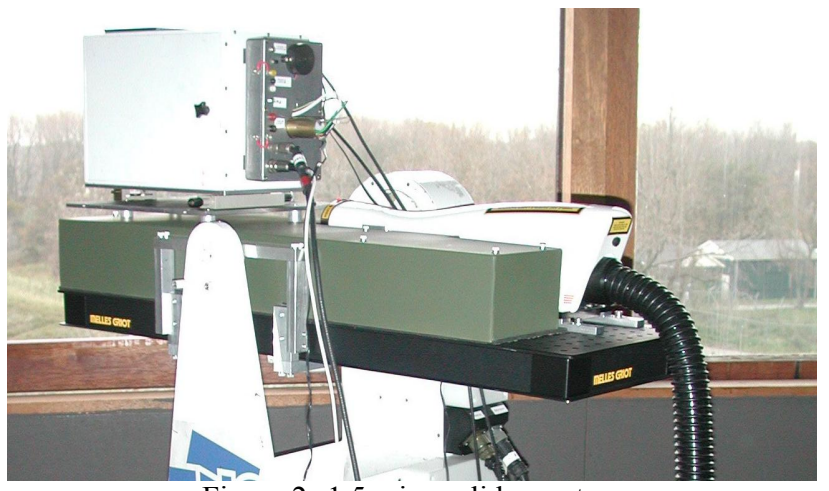

Figure 21.5 micron lidar system

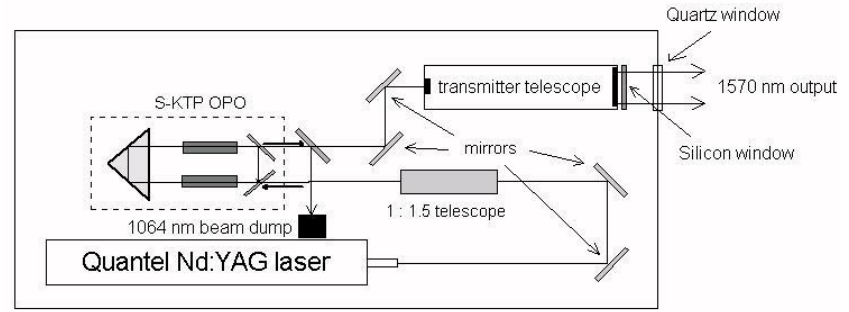

Figure 3 Optical layout of eye safe lidar transmitter.

We have made an OPO design that maximizes the non-linear conversion efficiency by using two crystals instead of one, and by using S-KTP instead of KTP crystals which has a higher damage threshold. We ended up with a ring cavity design. The OPO laser is connected to an optical telescope and in addition some filters are placed in the beam line to completely block the eye-hazardous laser pump light. The layout of the transmit section of the LIDAR is shown in Figure 3. The LIDAR system was operated with maximum pump pulse energy of $205 \mathrm{~mJ}$ at a rep-rate of $10 \mathrm{~Hz}$. The output from the transmitter at $1570 \mathrm{~nm}$ was around $30 \mathrm{~mJ}$.

The receiver is constructed using a $750 \mathrm{~mm}$ telescope, with an optical aperture of $125 \mathrm{~mm}$ diameter. An InGaAs APD detector with a diameter of $80 \mu \mathrm{m}$ was used. The small diameter APD detector is used because it allows a very high electrical bandwidth $(200 \mathrm{MHz})$, which is required for a small range resolution ( 0.3 meter). Figure 4 shows the results of the resolution measurements with two poles. At a separation of $60 \mathrm{~cm}$ the poles can still be distinguished. A resolution of $30 \mathrm{~cm}$ is achievable with some deconvolution if a higher sampling rate is used than shown in the figure.
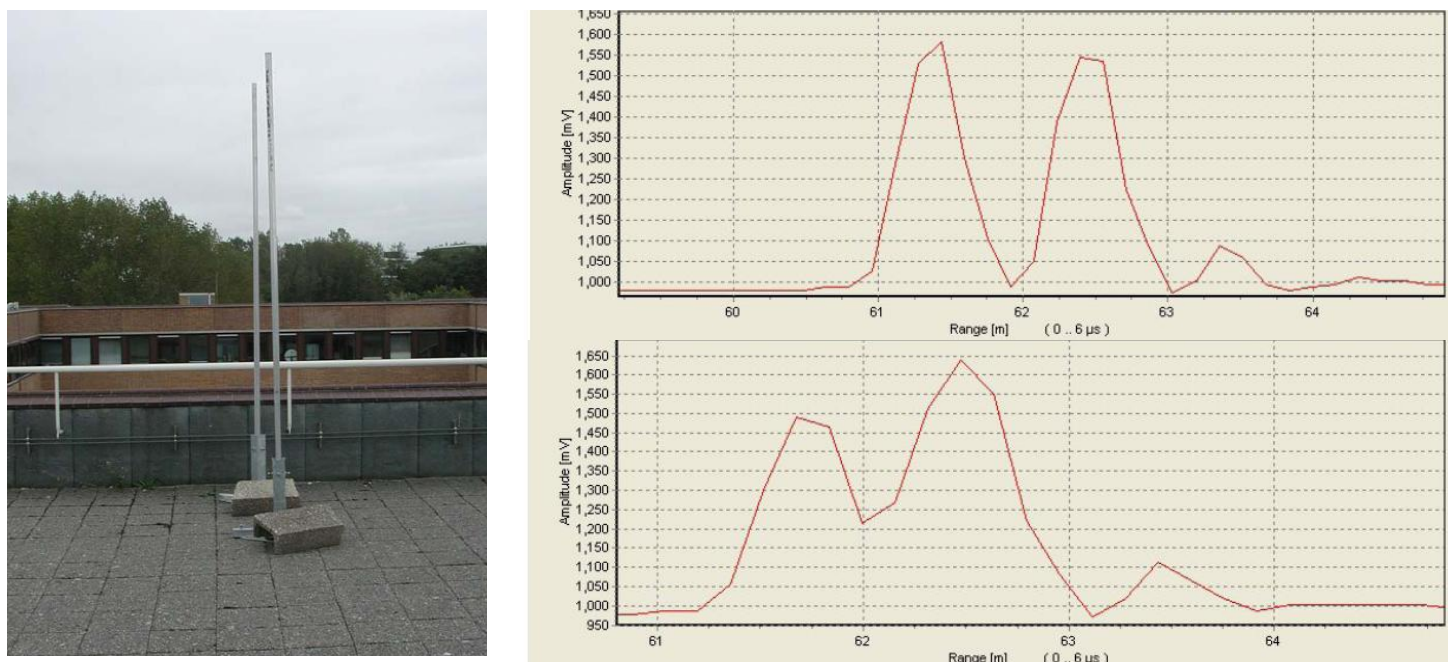

Figure 4 Resolution measurements with poles separated $100 \mathrm{~cm}$ (top right) and $60 \mathrm{~cm}$ (bottom right).

Figure 5 shows the laser range profile of a small sailing boat at a range of $3.4 \mathrm{~km}$. It is clear that the reflection from the sea surface is negligible. In addition, the signal-to-noise ratio is quite good. Therefore, in the simulations the sea surface 
return is not taken into account and the SNR is based on these results. In fact the noise is higher in the simulation to show the effect of noise more clearly.
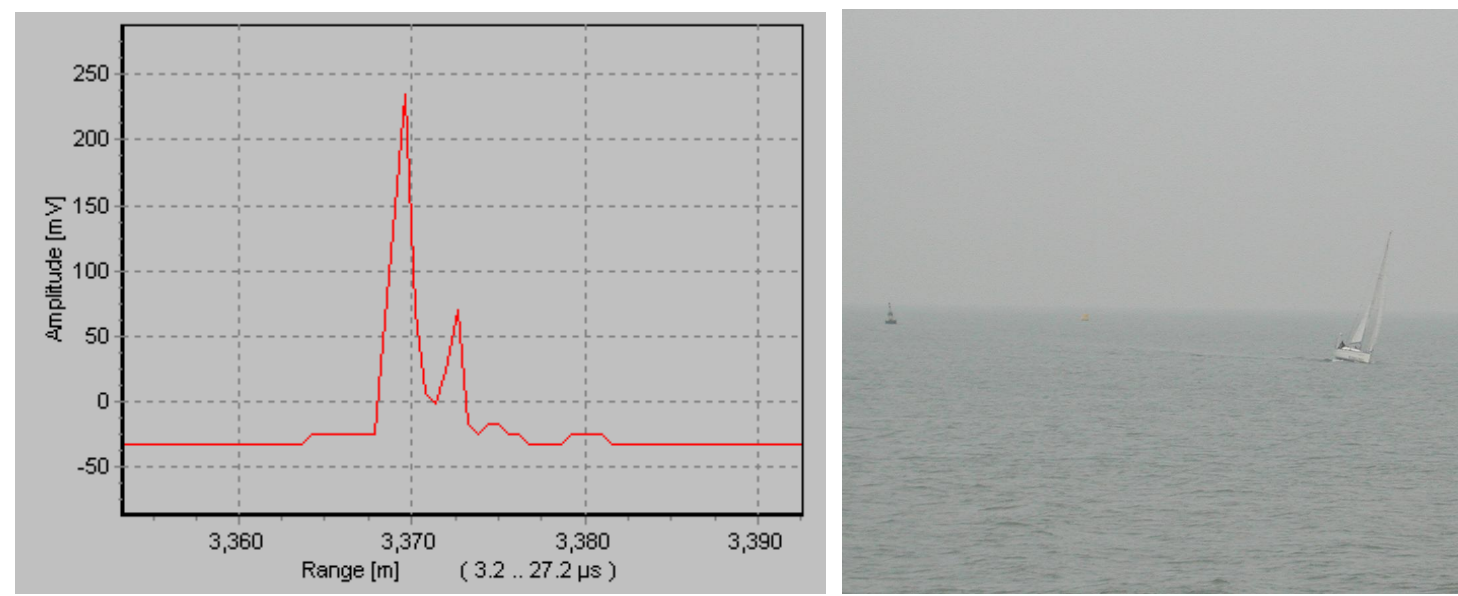

Figure 5 Laser range profile of a small sailing boat at $3.4 \mathrm{~km}$. The photograph was taken at short range.

\section{SIMULATED SENSOR DATA}

The data for the sensor are obtained through modeling where the targets are represented by so-called facet models. We have used facet models for three ships: a Boghammer, a cabin boat and a Rhib. The Boghammer is originally a high speed patrol boat designed by a Swedish company but is nowadays considered as typical for hostile ships that might be used by irregular groups in coastal waters. The cabin boat is a typical small civilian ship which can be found in littoral waters. The Rhib (Rigid hull inflatable boat) is a small fast boat which can transport several people and is typical for bridging the coast for larger ships that have to stay off-shore. They are often stored on board of military vessels, such as frigates, and are also used for life saving. In Figure 6 we show pictures of the ships that have been made on basis of the facet models and in Table 1 we summarize the main dimensions of the ships.
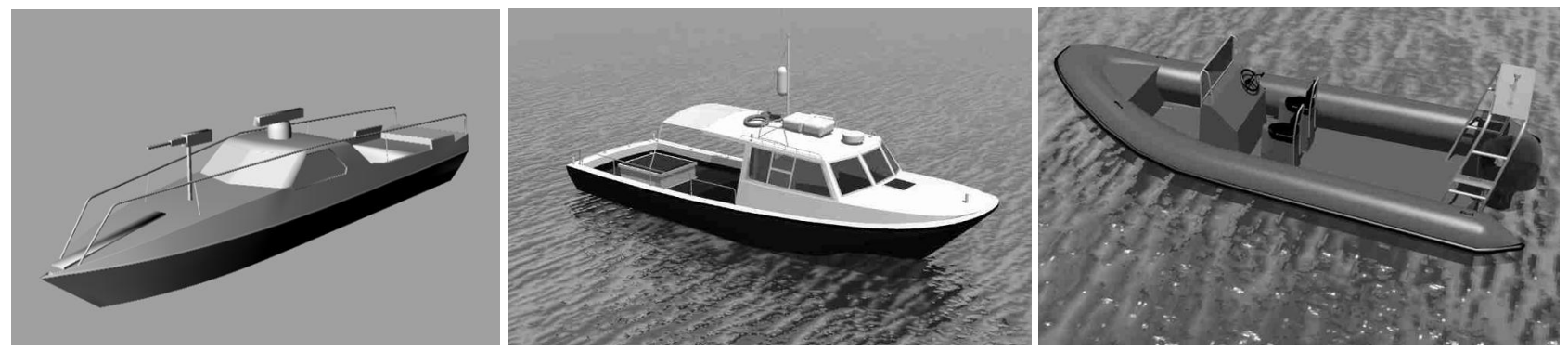

Figure 6 Pictures of the Boghammer (left), cabin boat (middle) and Rhib (right)

Table 1 The dimensions in meters of these targets.

\begin{tabular}{|l|l|l|l|}
\hline & length & width & height \\
\hline Boghammer & 12.8 & 2.7 & 3.0 \\
\hline Cabin boat & 10.5 & 3.3 & 3.7 \\
\hline Rhib & 6.8 & 2.6 & 1.0 \\
\hline
\end{tabular}

For the laser range profiler, simulated range profiles are obtained from an in-house electro-optics model (EOSTAR ${ }^{6}$ ) and the target facet models. Diffuse Lambertian reflection was assumed for all surfaces. ${ }^{3,4,5}$ The effect of diffuse reflection and the receiver noise was calculated in Matlab. The reflection from the sea surface was assumed negligible, which has been confirmed by several experiments in the past near the coast. ${ }^{7}$ Some of these results were shown in the previous section. 
From the scenario observation parameters like distances, and angles for elevation and azimuth are extracted. Using abovementioned models, simulated sensor data are obtained for the laser range profiler observing the three targets. Contributions from the sea surface are ignored. Figure 7 shows the simulated laser range profiles for the three ships according to the scenario.

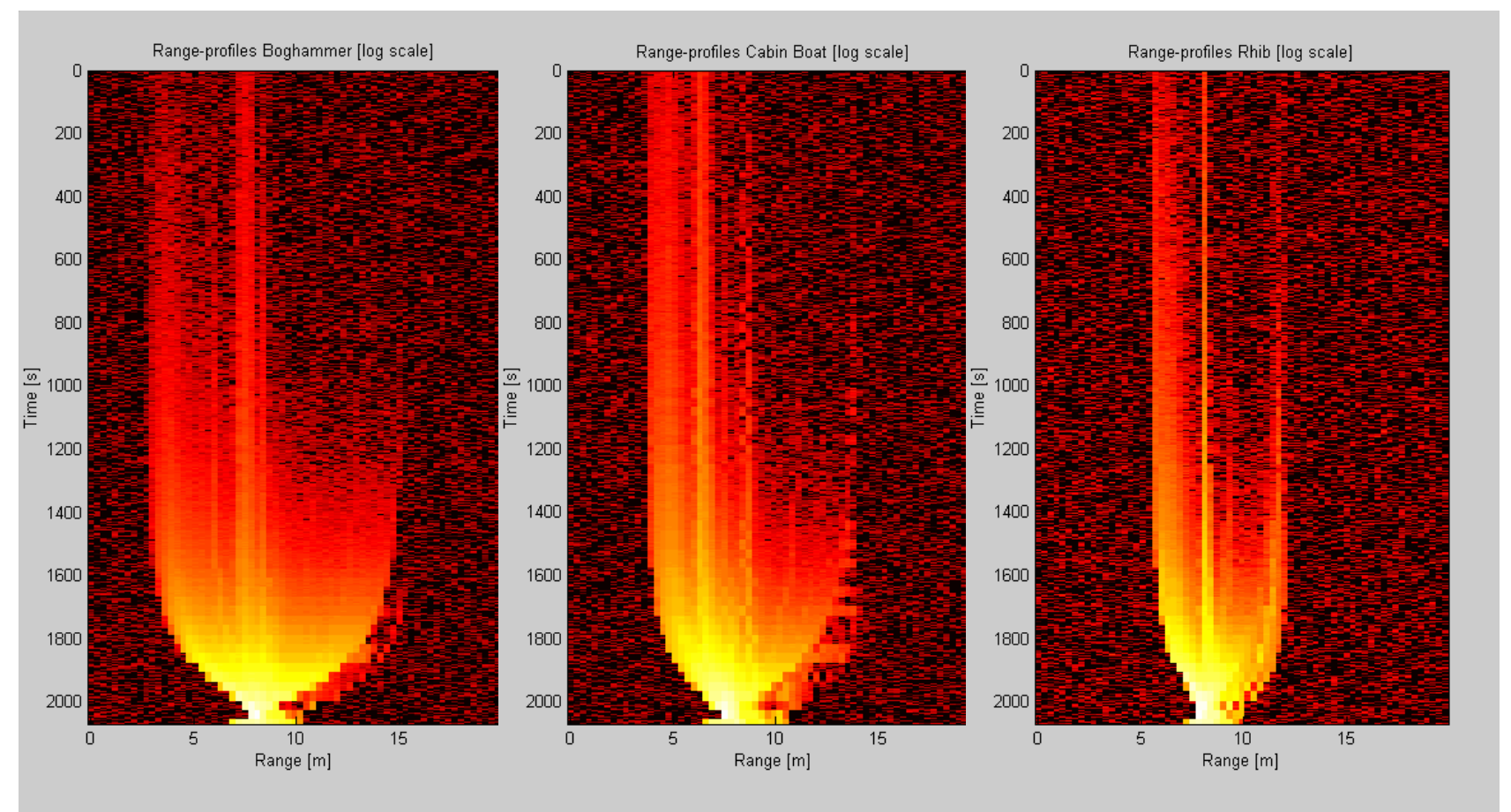

Figure 7 Simulated laser range profiles for the complete scenario with Boghammer (left), cabin boat (middle), and Rhib (right).

These laser range profiles have $30 \mathrm{~cm}$ range resolution. Noise has been added so that the signal at large distances (more than $6 \mathrm{~km}$ ) is dominated by noise. In Figure 7 the range profiles are plotted in the horizontal direction while the vertical axis indicates the time. The relation of time versus target distance or target aspect angle was shown in Figure 1. The range profiles show clearly the bow (left) and the cabin (middle). Note that the range profiles become strong and short in the bottom of figure 3.2, which corresponds to the end of the scenario. In this part of the scenario the ship is close to the coast and observed with azimuth angles near 90 degrees (i.e. line-of-sight perpendicular to the long axis of the ship) causing short and bright range profiles.

To classify the targets, the simulated data according to the scenario have to be compared with data in a database. Therefore, data for the databases have also been simulated using the model. The database consists of laser range profiles of the three ships for each 5 degrees of azimuth angle. Roll and pitch of the ships is not taken into account for the database. In the actual application of a laser range profiler in a coastal surveillance scenario the profile would be measured instead of simulated. However, the database might still consist of simulated range profiles since these would be much easier to obtain than experimental ranges at many aspect angles.

\section{CLASSIFICATION RESULTS}

For identification, we use correlation of laser range profiles to compare sensor data with data in the database. Since the aspect angle of the ship is known from the radar track data, the simulated "measured" laser range profile is correlated with the closest range profile (in aspect angle) in the database. Before correlation the "measured" laser range profile (LRP) is scaled to the database LRP: 


$$
L_{M}^{\prime}=S_{L} L_{M} \text { with } S_{L}=\frac{\sum L_{D B}}{\sum L_{M}},
$$

where $L_{M}$ is the "measured" LRP, $L_{D B}$ is the database LRP, and $S_{L}$ is the scaling factor. The maximum correlation is an indication whether the target corresponds to the target in the database:

$$
C_{M, D B}=\max \left[\sum L_{M}^{\prime}(\Delta x) L_{D B}\right] .
$$

This indication whether the target corresponds to the target in the database is expressed as a likelihood based on the correlation and the noise in the data:

$$
P=\exp \left[-F_{T} \frac{\left|\sum L_{M}^{\prime 2}+\sum L_{D B}^{2}-2 C_{M, D B}-N_{M} \sigma^{2} S_{L}^{2}\right|}{\sqrt{2 N_{M}} \sigma^{2} S_{L}^{2}}\right],
$$

where $F_{T}$ is a threshold factor, $N_{M}$ is the number of entries in the database LRP, and $\sigma$ is the noise in the "measured" LRP.

Figure 8 shows these likelihoods as images with the likelihoods plotted on a color scale. The "measured" LRP's for the range of aspect angles between 0 and 360 degrees are based on the Boghammer database with added noise. As expected, the figure on the left for the Boghammer shows that the "measured" and database LRP correspond very well for identical azimuth angle. Note that the negative azimuth angle (or $360 \mathrm{deg}$ minus azimuth) also agrees quite well. This is due to the almost symmetrical shape of the Boghammer. Likelihoods for the cabin boat and Rhib are about 2 orders of magnitude smaller, since their LRP's do not correlate very well with the "measured" LRP's from the Boghammer. However, if we increase the noise, the likelihoods of these boats increase considerably as we shall show next.
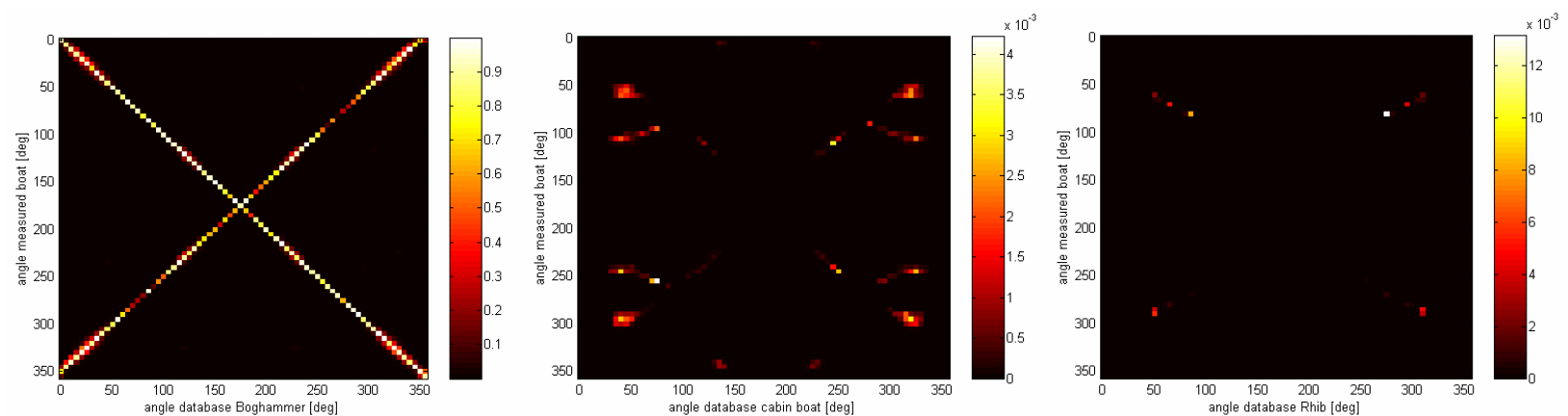

Figure 8 Likelihoods based on correlation of a "measured" LRP at various azimuth angles with database LRP's of Boghammer (left), cabin boat (middle), and Rhib (right).

Figure 9 shows the correlation and likelihood results for an approaching Boghammer. At the start of the scenario, the Boghammer is at long range and the signal-to-noise ratio is low. All three ships have low correlation and high likelihood. Due to the large noise in the LRP's it is not possible to identify the approaching ship as a Boghammer. After 15 minutes $(900 \mathrm{sec}$ ), the Boghammer is at a range of $6 \mathrm{~km}$ (see Figure 1) and the likelihood of the Boghammer exceeds those of the other two ships. Near the end of the scenario, the identification of the Boghammer fails. This is due to the side view of the ship. In side view the LRP's of the three ships are short and a more or less equal. 

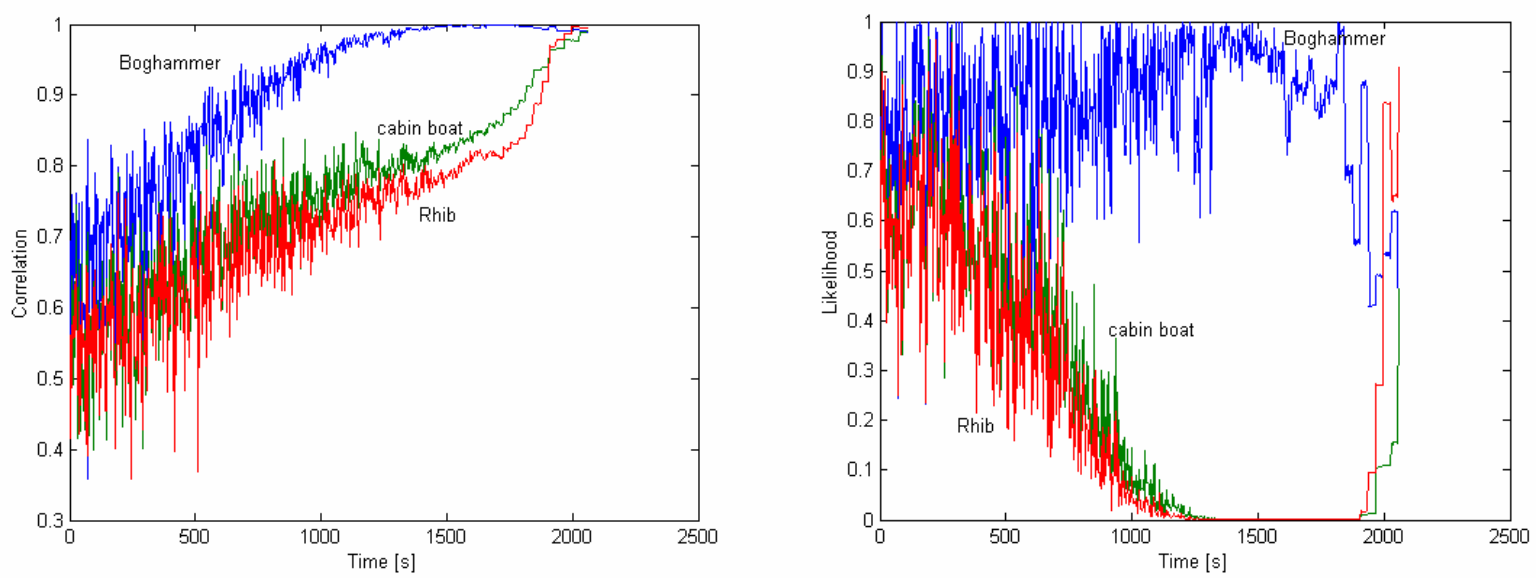

Figure 9 Correlation (left) and likelihood (right) for the three ships as a function of time in the scenario given an approaching Boghammer.

The three correlations (for the three ships) give three likelihoods when taking the noise into account. A moderate correlation with large noise gives a high likelihood but a low one for little noise. These likelihoods are used to obtain probabilities using Bayes theorem. In the Bayes classification, we want to obtain the a-posteriori probability $P\left(A \mid L_{M}\right)$ that a class $A$ is present given a measured laser range profile $L_{M}$.

$$
P\left(A \mid L_{M}\right)=\frac{P\left(L_{M} \mid A\right) P(A)}{P\left(L_{M}\right)},
$$

where $P\left(L_{M} \mid A\right)$ is the likelihood given by the correlation, $P(A)$ is the a-priori probability that class $A$ occurs, and $P\left(L_{M}\right)$ is a normalization factor. This normalization factor determines that the sum over all classes of these a-posteriori probabilities is one.

A target class "other" has to be used for the case that an LRP is measured of a boat that is not in the database. The problem is that $P\left(L_{M} \mid\right.$ other $)$ can not be determined from correlation of the LRP. In order to obtain the class "other" when all boats in the database give a low likelihood, we use a constant small value $\varepsilon$ for $P\left(L_{M} \mid\right.$ other $)$. To limit the number of free parameters, the a-priori probabilities will be set the same for all classes, effectively removing them. Now the normalization factor is given by

$$
P\left(L_{M}\right)=\sum_{\substack{\text { classes in } \\ \text { database }}} P\left(L_{M} \mid A\right)+\varepsilon,
$$

Figure 10 shows the result of the Bayesian classification. The figure on the left is quite similar to the likelihoods of Figure 9, which is to be expected since all a-priori information is discarded. Again the Boghammer is identified at shorter ranges with a maximum probability of $95 \%$ after $1200 \mathrm{sec}$, corresponding to $4 \mathrm{~km}$. The remaining $5 \%$ is claimed by the "other" class which is the consequence of the small value $\varepsilon$ of $5 \%$ for the likelihood of the "other" class $P\left(L_{M} \mid\right.$ other $)$. The figure on the right shows the effect of the other class. Here the Boghammer is removed from the dataset, while the scenario sensor data remain the same. Since the Rhib and the cabin boat do not match the scenario data, the "other" class is selected with $100 \%$ certainty. 

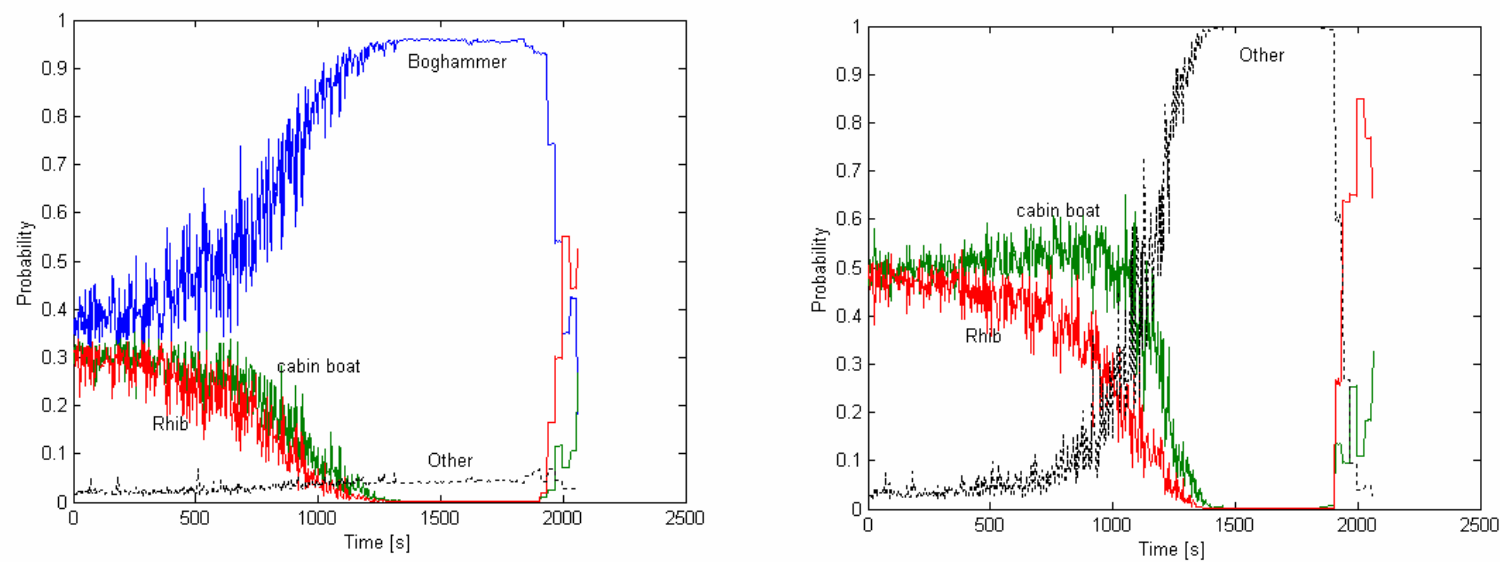

Figure 10 Bayes probabilities for an approaching Boghammer in the scenario. The figure on the right has the Boghammer removed from the database.

\section{DISCUSSION}

For the classification of the ships a Bayesian approach was followed. A priori information was not used in the analysis above, however, it could be used in the described method to improve the performance and to combine with information from other sensors or source. For instance, a priori information can be derived from expectation based on historical records, but can also come from AIS information or by inspection of observed characteristics. For example when a ships of small dimensions has a relative slow variation in elevation angle with respect to the waves, this can be an indication for excessive load, which can be considered as anomalous behaviour. Also a ship with a high speed can pre-select certain ship classes, so that a priori chances have to be adapted.

In practice to determine a priori probabilities we first have to determine which class of ships are relevant. In the scenario we have considered as relevant class all boats with a small dimension $(<15$ meter). All ships larger than 15 meter will not be considered. Note that the laser range profiler can also be used to determine the size of the boat.

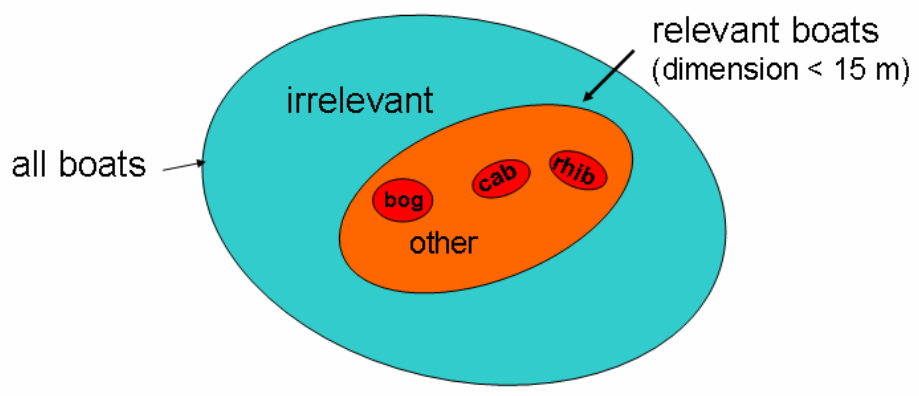

Figure 11 Schematic representation of the target classes

In the presented method there was no learning or averaging of laser range profiles. Learning could be achieved by adjusting the a priori information. When on basis of a priori information a classification result is obtained, this information can be used to adjust the a priori probabilities for a following classification. This is done by adding classification results in the next classification cycle. The a priori probabilities are adjusted with a 'coupling' factor to the classification results which is small $(<1 \%)$ so that fluctuations in the classification results due to low quality observations will not influence the final outcome too much. In Figure 12 this learning cycle is visualized. 


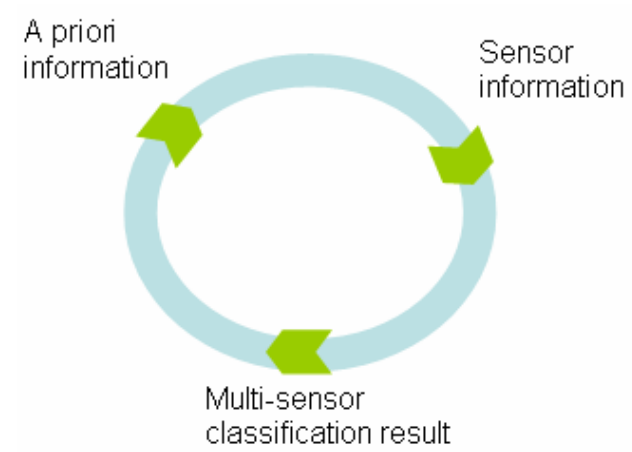

Figure 12 Schematic representation of the learning cycle.

\section{SUMMARY AND CONCLUSIONS}

We have presented a concept for coastal surveillance using a laser range profiler for identification of sea-surface targets. This concept has been evaluated and demonstrated using modeled sensor data. It is shown that the Bayesian classification gives good identification results when the signal-to-noise ratio is not too low. The experimental results confirmed the simulation assumptions of good range resolution and small sea-surface clutter.

An important factor is the database. For good classification results a detailed database is needed for all ships which are of interest. In practice it will be difficult to obtain such a database by measurements alone. An option is to use modeling results; however, this has to be checked by experiments.

\section{REFERENCES}

1. R. van der Heiden, Aircraft Recognition with Radar Range Profiles, Thesis University of Amsterdam, 1998.

2. R.S. Berkowitz, Modern Radar - Analysis, Evaluation, and System Design, John Wiley \& Sons, Inc., 1965.

3. J.J. Koenderink, A.J. van Doorn, "Bidirectional Reflection Distribution Function of Thoroughly Pitted Surfaces", Int. J. Comp. Vision 31(2/3), 129-144 (1999).

4. P. Beckmann, A. Spizzichino, The scattering of Electromagnetic Waves from Rough Surfaces, Pergamon Press, 1963.

5. K. Torrance, E. Sparrow, "Theory for off-specular reflection of roughened surfaces", J. Opt. Soc. Am. 57, 11051114 (1967).

6. Kunz, G.J., et al., "Status and developments in EOSTAR, a model to predict IR sensor performance in the marine environment", Proc. SPIE Vol. 5572-12, pp. 1-12 (2004).

7. Kunz, G.J., et al., "Detection of small targets in a marine environment using laser radar", Proc. SPIE Vol. 5885 (2005). 